\title{
DESIGN AND FABRICATION OF THE TRAPEZOIDAL ELECTROSTATIC COMB-DRIVE ACTUATOR
}

\author{
Pham Hong Phuc ${ }^{1}$, Dinh Khac Toan ${ }^{1}$, Dang Bao Lam ${ }^{1}$, \\ Nguyen Tuan Khoa ${ }^{1}$, Nguyen Tien Dung ${ }^{2}$ \\ ${ }^{1}$ Hanoi University of Science and Technology, Vietnam \\ ${ }^{2}$ Thai Nguyen University of Technology, Vietnam
}

\begin{abstract}
This paper reports the design, fabrication and characterization process of the trapezoidal Electrostatic Comb-drive Actuator (ECA) with the slope angle $\alpha=2^{\circ}$. Together with the trapezoidal ones, the rectangular ECA with identical dimension was also designed and fabricated for comparison purpose. In order to reduce calculating deviation, the fringing effect was also taken into consider while carrying out theoretical analysis. The obtained results pointed out the fact, that the trapezoidal ECA excels the rectangular ones with the same numbers of teeth in electrostatic force and displacement generation, while requires relatively low driving voltage. But it is also observed that with higher driving voltage (larger than $50 \mathrm{~V}$ ), the trapezoidal ECA starts to lose its stability (the lateral pull-in phenomenon occurs).
\end{abstract}

Keyword: electrostatic comb-drive actuator.

\section{INTRODUCTION}

A rectangular electrostatic comb-drive actuator (ECA) was firstly introduced by Tang et al. [1]. While working, the fabrication errors can cause imbalance of the forces acting on the electrodes, which can lead to destabilization of the rectangular ECA [2]. Impact of destabilization increases with a thinner finger thickness and lower stiffness of the beam of the actuator. On the other hand, for overcoming this problem, choosing larger size of the electrodes could also lead to bulky dimension of the whole system. In [3], Johan B. C. Engelen et al. mentioned about the optimized shape of the ECAs. After optimization, the ECA can generate larger and constant electrostatic force. With the same number of fingers in a length unit, the ECA with optimized teeth can work with relatively high stability, good vibration damping ability, and can produce the forces, which is 1.8 larger than those generated by the normal rectangular ECA.

Since the first research of an ECA by Tang et al [1], there have been a large number of applications of this type of actuators [4]. One of them was a linear motor [5] using the linear ECAs, the other one was a rotational motor $[6,7]$ with the curved ECA. The ECAs can also be found in the micro transportation and assembling systems $[8,9,10]$. In those applications, the actuating members in the ECA are tangential electrostatic forces, which are relatively small. The normal electrostatic forces, which are much larger, can also 
be used e.g. in the gap-closing actuator (GCA) [5], etc. However, those actuators using the normal electrostatic force have to deal with a disadvantage that the normal force could rapidly decrease when the distance between the fingers of ECA becomes larger. The rectangular ECAs using tangential forces have the simple structure, simple fabrication process, and high stability, but they are still accompanied with low overall performance characteristics such as small force at relatively high driving voltage and large dimension. To overcome those disadvantages, the authors present the ECA with the trapezoidal finger, in which the combination of the tangential and normal electrostatic forces will be used as a driving force.

\section{THE TRAPEZOIDAL ECA}

\subsection{ECA design}

Fig. 1 shows the configuration of the trapezoidal ECA with following parameters: slope angle $\alpha=2^{\circ}$, initial gap between two opposite finger $d$, overlap $a$, tooth thickness $b$ and tooth length $l_{c}$. We know that when the slope angle $\alpha$ increases, the electrostatic

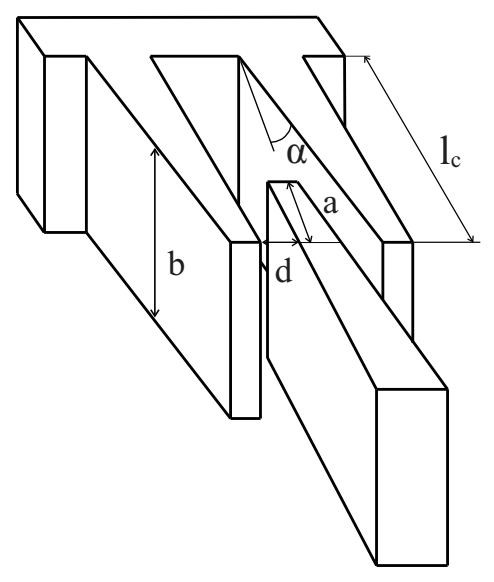

Fig. 1. Configuration of the trapezoidal ECA

forces acting on the fingers also increase, but the gained displacement will decrease, and vice versa. While the trapezoidal ECA is working, the overlap becomes larger, the gap $d$ becomes smaller, and therefore, the acting forces in the ECA increase simultaneously.

\subsection{Edge effect (fringing effect)}

When two plates are applied by $V$-voltage between (one plate is positive and the other is negative) as shown in Fig. 2, an electric field is created between them. The electric field lines seem linear in the center and non-linear toward the edge of the capacitor. This phenomenon changes its capacitance, therefore, it varies forces applying on two plates. According to Guo Zhanshe et al. [11], the capacitance $C$ can be expressed as:

$$
C=\frac{a b \varepsilon \varepsilon_{0}}{d}+\frac{b \varepsilon \varepsilon_{0}}{\pi}\left\{1+\ln \left[1+\frac{\pi a}{d}+\ln \left(1+\frac{\pi a}{d}\right)\right]\right\}+\frac{a \varepsilon \varepsilon_{0}}{\pi}\left\{1+\ln \left[1+\frac{\pi b}{d}+\ln \left(1+\frac{\pi b}{d}\right)\right]\right\}
$$


where $d$ is the gap between two fingers, $a$ is the overlap of those and $b$ is the thickness of a comb finger.

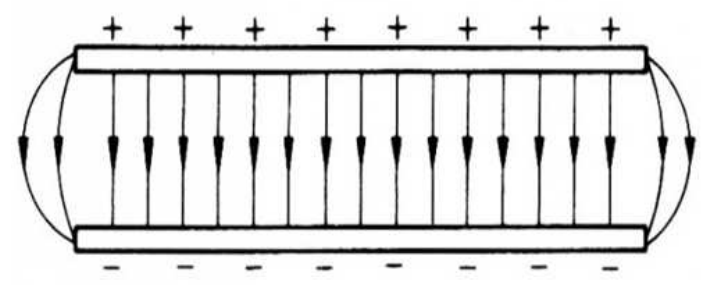

Fig. 2. Electric field between two parallel plates

Fig. 3a presents the force analysis in the well-known rectangular ECA, in which the acting tangential force $F_{y}$ and normal force $F_{x}$ can be expressed as below:

$$
F_{x}=\frac{1}{2} \frac{\partial C(d)}{\partial d} V^{2}, \quad F_{y}=\frac{1}{2} \frac{\partial C(a)}{\partial a} V^{2}
$$

\subsection{Force analysis in the trapezoidal ECA}

In Fig. 3b, the initial position of a point on the surface of trapezoidal tooth is at $\mathrm{A}_{0}$. After applying a driving voltage, the movable tooth is pulled in y-direction, thus, it

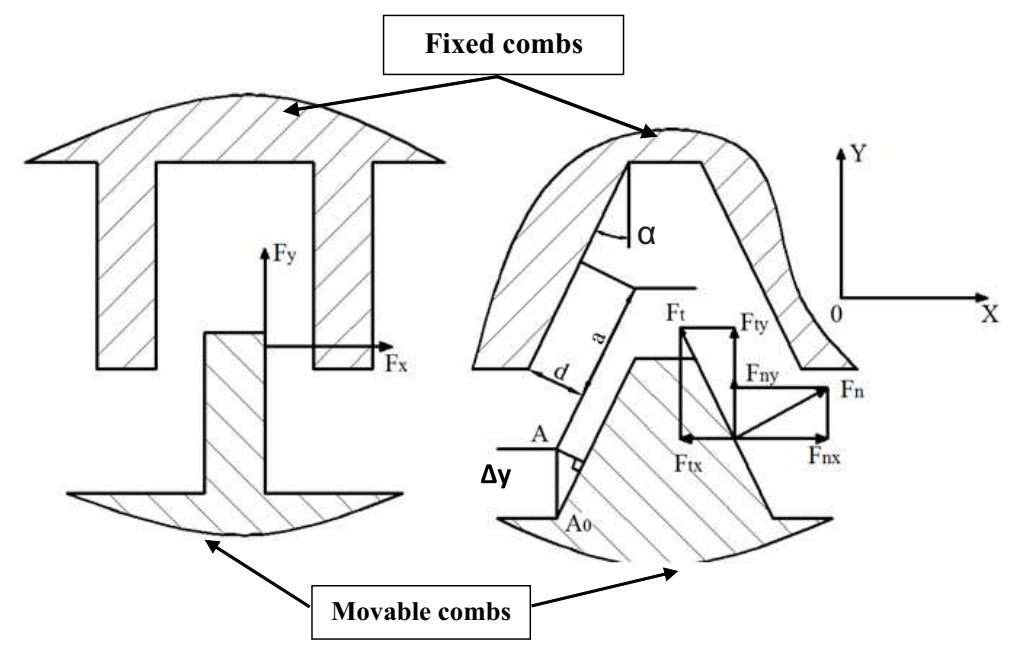

a) Forces in the rectangular ECA b) Forces in the trapezoidal ECA

Fig. 3. Force analysis in the comb actuator

is moved a distance of $\Delta y$, and the point $\mathrm{A}_{0}$ travels to the point $\mathrm{A}$. Therefore, the gap $d$ and the overlap $a$ are calculated as:

$$
d=d_{0}-\Delta y \sin \alpha, \quad a=a_{0}+\Delta y \cos \alpha
$$


As the rectangular ECA, the trapezoidal ECA is acted by the tangential force $F_{t}$ and the normal force $F_{n}$. Those are calculated in Eq. (2), where the gap $d$ and the overlap $a$ are defined by Eq. (3). Fig. $3 \mathrm{~b}$ shows that the force $F_{t}$ is separated into $F_{t x}$ in $x$-direction and $F_{t y}$ in $y$-direction, and similarly, $F_{n}$ is separated into $F_{n x}$ and $F_{n y}$. as below:

Total forces acting on the one side of the finger in $x$ and $y$ direction can be calculated

$$
\sum F_{y}=F_{t y}+F_{n y}, \quad \sum F_{x}=F_{n x}-F_{t x}
$$

Or

$$
\sum F_{y}=F_{t} \cos \alpha+F_{n} \sin \alpha \text { and } \sum F_{x}=F_{n} \cos \alpha-F_{t} \sin \alpha
$$

Where parameters of the trapezoidal ECA were chosen as follows: the initial overlap $a_{0}=5 \mu \mathrm{m}$, the initial gap $d_{0}=2 \mu \mathrm{m}$, the angle $\alpha=2^{\circ}$. The dimensions of the rectangular ECA are intentionally chosen as the same as those of the trapezoidal ECA.

From Eqs. (1), (2) and (4), the relation between the forces acting in the ECA and the moved distance $\Delta y$ can be established in Fig. 4a. The ratio of the force in the trapezoidal ECA to the force in the rectangular ECA is shown in Fig. $4 \mathrm{~b}$.

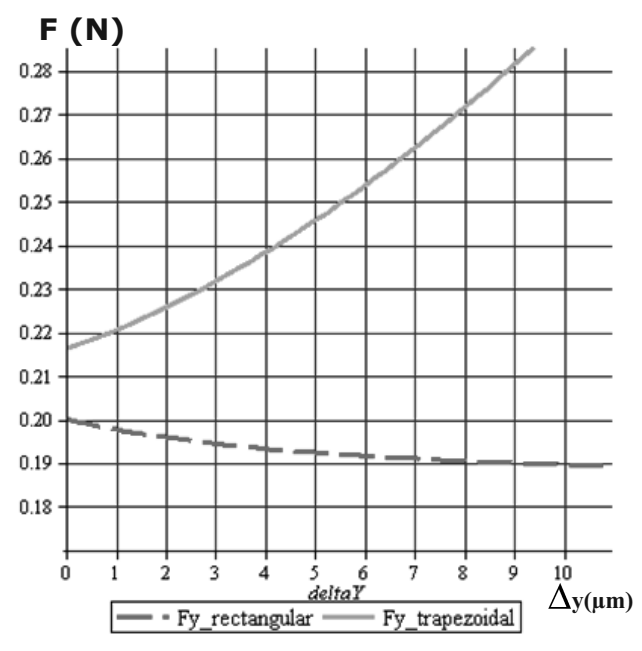

(a) Force - displacement relation

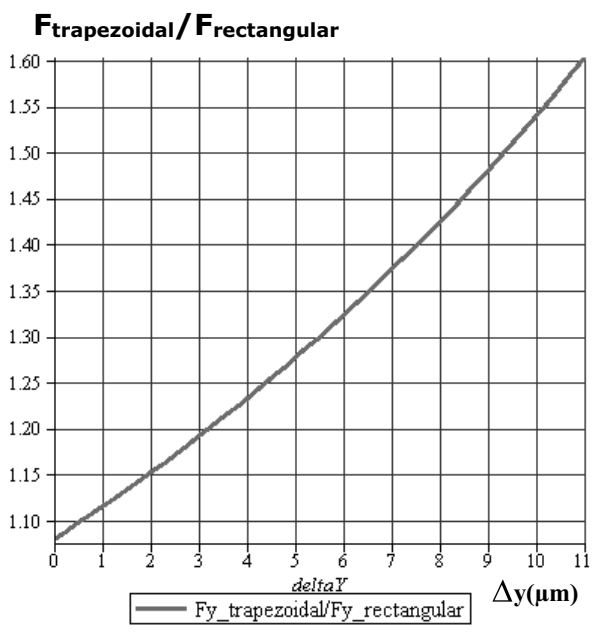

(b) The ratio of forces

Fig. 4. Effects of the ratio $R / h\left(m=1, n=6, k=k_{1}=1, \mathrm{~T}=300 \mathrm{~K}\right.$, $\left.\xi_{*}=0.1, L / R=1\right)$

It is observed that when $\Delta y$ increases, the force in the rectangular ECA decreases, but on the contrary, the force in the trapezoidal ECA increases rapidly. The ratio of two forces also increases as presented in Fig. $4 \mathrm{~b}$ and this ratio approximately equals 1.55 when $\Delta y=10 \mu m$. 


\subsection{Displacement in the trapezoidal ECA}

Fig. 5a shows the designed trapezoidal ECA which following parameters: the length of the beam $l=700 \mu \mathrm{m}$, the rectangular section $b_{w} \times h=4 \times 30 \mu \mathrm{m}^{2}$, number of movable fingers $n=134, E=169 \mathrm{GPa}$ is Young's modulus of single crystalline silicon. Fig. 5b presents the calculation diagram, where the forces acting on beam $n F_{t}$ are replaced by a

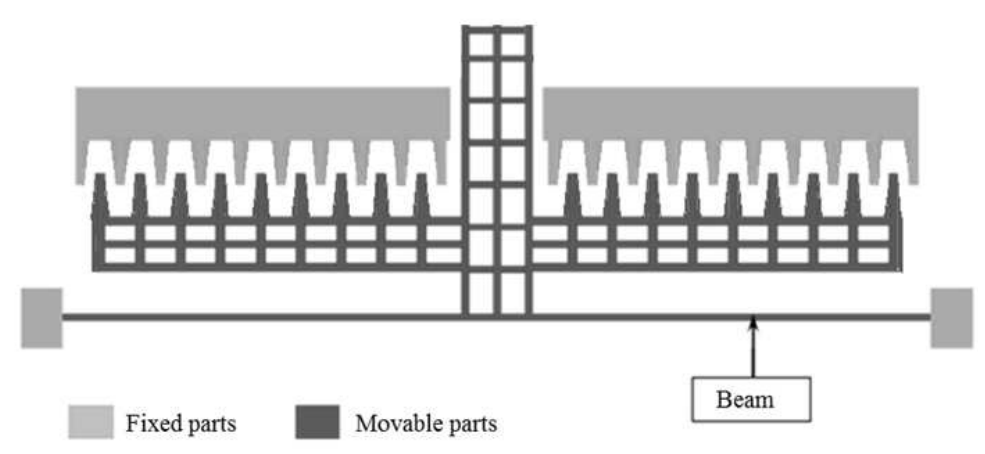

(a) Configuration of the trapezoidal ECA

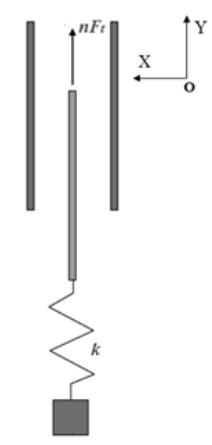

(b) Calculation diagram

Fig. 5. Force calculation in the trapezoidal ECA

spring with a stiffness of $k$. The stiffness $k$ is defined as:

$$
k=\frac{4 E h b_{w}^{3}}{l^{3}}
$$

Replacing parameters in Eq. (6) by their values, we get the value of beam stiffness $k=3.7845 \mu \mathrm{N} / \mu \mathrm{m}$. Because the viscous drag force and friction force are negligible while the ECA is working, the equation of force balance of the actuator can be presented as below:

$$
n F_{t}(\Delta y)-k \Delta y=0
$$

Table 1. Calculated displacements of the trapezoidal ECA

\begin{tabular}{|c|c|c|c|c|}
\hline Voltage $(\mathrm{V})$ & 20 & 30 & 40 & 50 \\
\hline Displacement $(\mu \mathrm{m})$ & 1,25 & 2,95 & 5,69 & 10,57 \\
\hline
\end{tabular}

We use the numerical method to solve Eq. (7) with two steps: Firstly estimating initial roots by geometry method, and then using Newton-Raphson method to solve Eq. (7) to get value of displacement of the trapezoidal ECA. The results are shown in Tab. 1.

\subsection{Simulation of displacements of the trapezoidal ECA}

The rectangular and trapezoidal ECA are simulated in multi-field (electric and mechanical fields) using FEA method (Finite Element Analysis). Fig. 6 shows a result from simulation of the trapezoidal ECA working at voltage of $50 \mathrm{~V}$ and with the number 


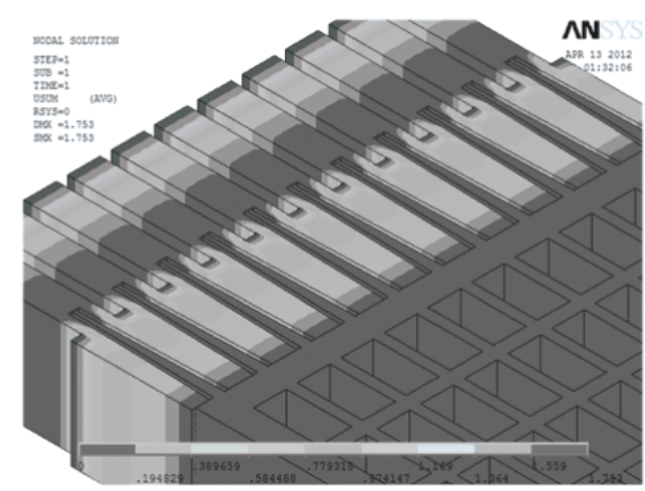

Fig. 6. Displacement of the trapezoidal ECA at $50 \mathrm{~V}$ (11 comb fingers)

Table 2. Simulated displacements of the trapezoidal ECA

\begin{tabular}{|c|c|c|c|c|}
\hline Voltage $(\mathrm{V})$ & 20 & 30 & 40 & 50 \\
\hline Displacement $(\mu \mathrm{m})$ & 1,32 & 3,16 & 6,15 & 10,81 \\
\hline
\end{tabular}

of comb fingers $n=11$. Simulation results with the driving voltages ranged from $20 \mathrm{~V}$ to $50 \mathrm{~V}$ are presented in Tab. 2.

\section{FABRICATION AND CHARACTERIZATION}

\subsection{Fabrication}

The ECAs has been fabricated by using SOI wafer with the thicknesses of the device layer, buried $\mathrm{SiO}_{2}$ layer, and silicon substrate were $30 \mu \mathrm{m}, 4 \mu \mathrm{m}$ and $450 \mu \mathrm{m}$, respectively.

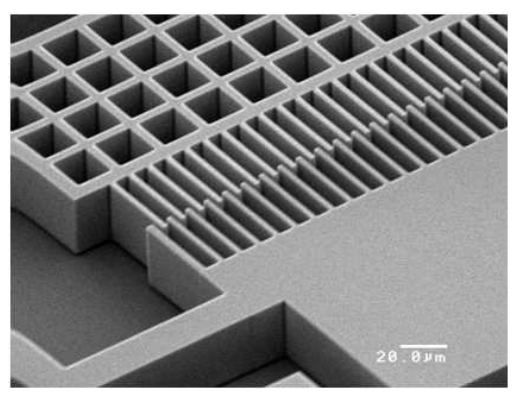

(a) Rectangular teeth

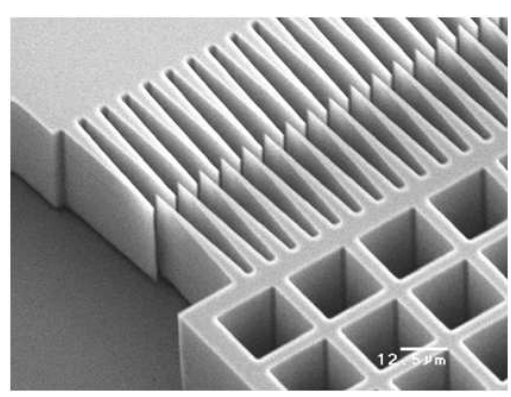

(b) Trapezoidal teeth

Fig. 7. SEM images of the rectangular and trapezoidal ECAs

The rectangular and trapezoidal ECAs after fabrication are shown in Fig. 7a and 7b, respectively. Fig. 8 shows the video-captured of the rectangular (8a) and trapezoidal (8b) 


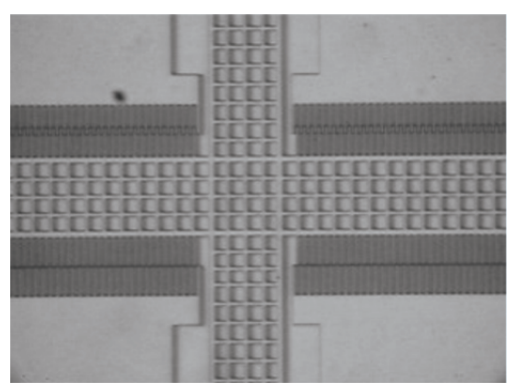

(a) Rectangular ECA

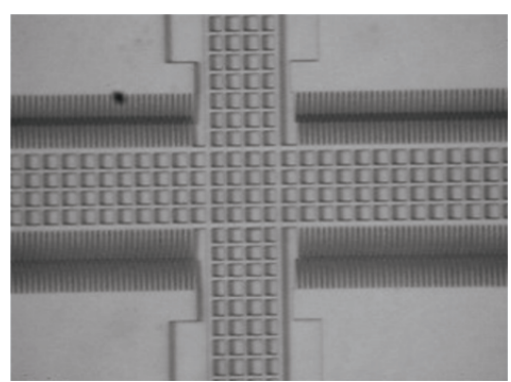

(b) Trapezoidal ECA

Fig. 8. The video-captured image when the ECAs working at $30 \mathrm{~V}$

ECA. It is clearly observed that at the same driving voltage $\left(V_{p p}=50 \mathrm{~V}\right)$, the displacements of the trapezoidal one is larger than those of the rectangular ECA.

To perform the operational test, the ECAs were driven by square wave periodic voltage ranged from $20 \mathrm{~V}$ to $50 \mathrm{~V}$. Fig. 9 shows the video-captured photograph taken

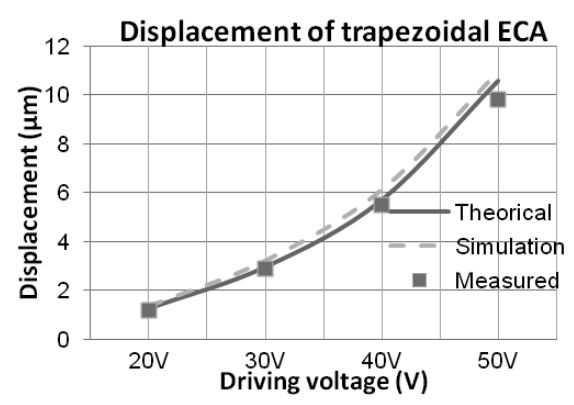

(a) Displacement - Driving voltage

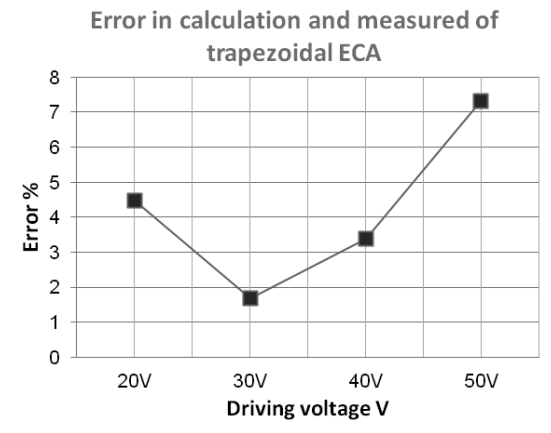

(b) Deviation between calculated and measured data

Fig. 9. Displacement of trapezoidal ECA

when measuring displacements of the rectangular and trapezoidal ECAs at the voltage of $30 \mathrm{~V}$. Tab. 3 shows the measured displacements at 20, 30, 40 and $50 \mathrm{~V}$, respectively.

Table 3. Measured displacements $(\mu \mathrm{m})$ of the rectangular and trapezoidal ECAs

\begin{tabular}{|c|c|c|c|c|}
\hline Finger/Voltage (V) & 20 & 30 & 40 & 50 \\
\hline Rectangular & 1 & 3 & 4 & 6 \\
\hline Trapezoidal & 1.5 & 3.5 & 7 & 10.5 \\
\hline
\end{tabular}

It is also observed that when driving voltage was increased to $60 \mathrm{~V}$, the lateral pull-in phenomenon occurs in the trapezoidal ECA. 


\subsection{Characterization}

Fig. 9a shows the relation between the calculated, simulated and measured displacements and the driving voltage of the trapezoidal ECA. With increased value of the driving voltage, the value of displacement also increases and a kind of quadratic relation is created. The largest deviation between the calculated data and the measured ones is close to $8 \%$ at the voltage of $50 \mathrm{~V}$ as shown in Fig. 9b.

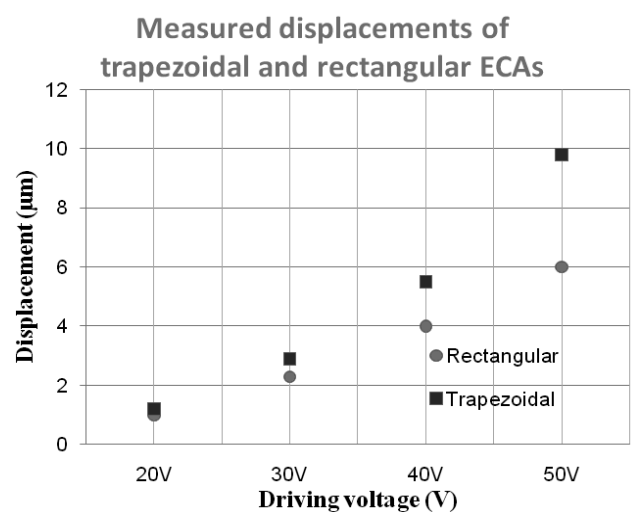

(a)

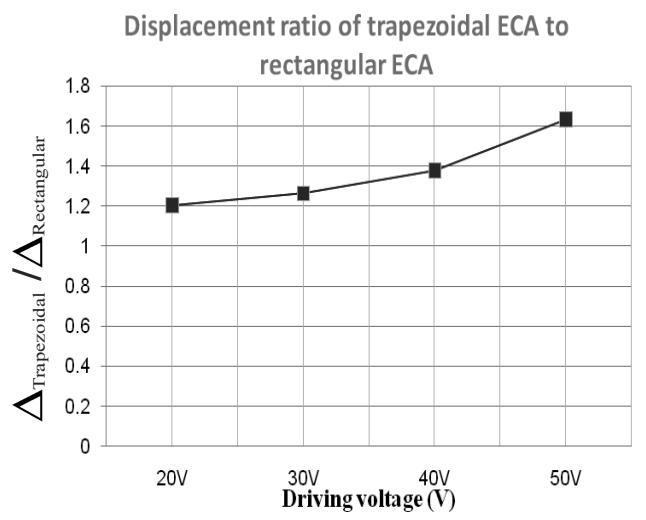

(b)

Fig. 10. Relation between measured displacements and driving voltage (a); the displacement ratio (b) of the rectangular and trapezoidal ECAs

The graph comparing measured displacements in the rectangular and trapezoidal ECAs is shown in Fig. 10. At low voltages, there is almost no deviation between both types of ECAs. When driving voltage increases (from $40 \mathrm{~V}$ to $50 \mathrm{~V}$ ), the measured displacements of the trapezoidal ECA are much larger than those of the rectangular ones. At voltage of $50 \mathrm{~V}$, this ratio reaches the value of 1.62 .

\section{CONCLUSION}

This paper has presented the design, fabrication and testing of both types of the ECAs, i.e. rectangular and trapezoidal. In comparison with the classical rectangular ECAs, the trapezoidal ones have several advantages and also minor disadvantage as below:

1. With the same teeth number, counted in the same length unit, the forces generated in the trapezoidal ECA is larger than those in the rectangular one. The attracting force in the movement direction increases 1.1 times initially, and 1.55 times at displacement of $10 \mu \mathrm{m}$.

2. The obtained results show that displacement of the trapezoidal ECA is larger the those of the rectangular ECA. The largest deviation $(62 \%)$ was observed at voltage of $50 \mathrm{~V}$. On the other hand, the measured values of displacement are smaller than the theoretically calculated and simulated ones, which can be explained by measurement and fabrication errors. 
3. When driving voltage increased to $60 \mathrm{~V}$, the lateral pull-in phenomenon occured because of the unbalanced state of the force components in $x$-direction.

\section{ACKNOWLEDGEMENT}

This work is supported by the project number: B2012.01.32, Ministry of Education and Training of Vietnam.

\section{REFERENCES}

[1] William C. Tang, Tu-Cuong H. nguyen, Michael W. Judy, Roger T. Howe, Electrostatic-comb Drive of Lateral Polysilicon Resonators, Sensors and Actuators, A21-A23, (1990), 328-331.

[2] Yang-Chen Chen, Ian Chao-Min Chang, Rongshun Chen, Max Ti-Kuang Hou, On the side instability of comb-fingers in MEMS electrostatic devices, Sensors and Actuators, A 148, (2008) 201-210.

[3] Johan B. C. Engelen, Leon Abelmann, Miko C. Elwenspoek, Optimized comb-drive finger shape for shock-resistant actuation, J. Micromech. Microeng., 20, (2010), 105003 (9pp), doi: 10.1088/0960-1317/20/10/105003.

[4] James J. Allen, MEMS applications, Taylor \&3 Francis Group, LLC, (2005).

[5] N. R. Tas, A. H. Sonnenberg, A. F. M. Sander, M. C. Elwenspoek, Surface Micromachined Linear Electrostatic Stepper Motor, Proceedings of International Solid State Sensors and Actuators Conference Transducers 97, 2, (1997), pp 777-780.

[6] Pham Hong Phuc, Dao Viet Dung, Bui Thanh Tung, Susumu Sugiyama, A micro rotational motor based on ratchet mechanism and electrostatic comb-drive actuators, Apcot, Tainan, Taiwan, (2008).

[7] Phuc Hong Pham, Dzung Viet Dao, Lam Bao Dang, Susumu Sugiyama, Single mask, simple structure micro rotational motor driven by electrostatic comb-drive actuators, J. Micromech. Microeng., 22, (2012).

[8] Pham Hong Phuc, Study on Micro Transportation Systems Based on Electrostatic Actuators Utilizing Micro Electro Mechanical Systems (MEMS) Technology, Ph.D Thesis, Ritsumeikan University, Japan, (2007).

[9] Pham Hong Phuc, Dao Viet Dung, Satoshi Amaya, Ryoji Kitada and Susumu Sugiyama, Straight movement of micro containers based on ratchet mechanisms and electrostatic combdrive actuators, Journal of Micromechanics and Microengineering, 16, (2006), p2532- 2538.

[10] Pham Hong Phuc, Dao Viet Dung, Satoshi Amaya, A micro transportation system (MTS) with large movement of containers driven by electrostatic comb-drive actuators, Journal of Micromechanics and Microengineering, 17, (2007), p2125-2131.

[11] Guo Zhanshe, Feng Zhou, Cao Le, Fan Shangchun, Theoretical and experimental study of capacitance considering fabrication process and edge effect for MEMS comb actuator, Microsyst Technol, DOI 10.1007/s00542-010-1162-1, (2010). 